\title{
Confort térmico y crecimiento de corderas West African pastoreando con y sin acceso a sombra artificial
}

\author{
López, R. ' ; Pinto-Santini, L. ${ }^{\circledR} ;$ Perozo, D. ${ }^{\text {; }}$ Pineda, J. ${ }^{\text {; }}$ Oliveros, I. ${ }^{2}$; Chacón, T. ${ }^{3}$; Rossini, M. ${ }^{3}$ y Ríos de Álvarez, L. ${ }^{1}$
}

'Instituto de Producción Animal. Facultad de Agronomía. Universidad Central de Venezuela. Maracay. Venezuela.

${ }^{2}$ Instituto Nacional de Investigaciones Agrícola (INIA). Campo Universitario. Maracay. Venezuela.

${ }^{3}$ Facultad de Ciencias Veterinarias. Universidad Central de Venezuela. Maracay. Venezuela.

\section{Palabras clave adicionales}

Estrés calórico.

Crecimiento.

Coeficiente de tolerancia al calor.

Ovinos.

Índice de masa corporal.

\author{
AdDITIONAL KEYWORDS \\ Heat stress. \\ Growth. \\ Heat tolerance coefficient. \\ Sheep. \\ Body mass index.
}

\section{INFORMACIÓN}

Cronología del artículo.

Recibido/Received: 20.5.2014

Aceptado/Accepted: 9.3.2015

On-line: 10.6 .2015

Correspondencia a los autores/Contact e-mail:

liviapintosantini@gmail.com

\section{RESUMEN}

Con la finalidad de evaluar el efecto de la presencia o no de sombra durante el pastoreo sobre el crecimiento y confort térmico de corderas mestizas West African, se realizó un estudio utilizando 24 hembras ovinas de $13,08 \pm 3,0 \mathrm{~kg}$ de peso vivo, las cuales fueron distribuidas al azar en dos tratamientos: SOMBRA: pastoreo en potrero de pasto estrella (Cynodon nlemfluensis) con acceso voluntario a sombra artificial o techo de lona de $36 \mathrm{~m}^{2}\left(3 \mathrm{~m}^{2}\right.$ por animal) y SOL: pastoreo en potrero de pasto estrella sin disponibilidad de sombra natural o artificial. La experiencia se realizó en el Laboratorio-Sección Ovinos de la Facultad de Agronomía - Universidad Central de Venezuela. Los animales se encontraban pastoreando en dos potreros de pasto estrella durante 6 semanas. Las mediciones realizadas fueron: mediciones climáticas tomadas con dos estaciones meteorológicas portátiles (una para cada potrero y la de sombra colocada debajo del techo) que registraban temperatura ambiente (TA, ${ }^{\circ} \mathrm{C}$ ), humedad relativa $(H R, \%)$ y radiación solar $\left(R S, w / \mathrm{m}^{2}\right)$, con estas mediciones se estimó el índice de temperatura y humedad o ITH. Temperatura corporal $\left({ }^{\circ} \mathrm{C}\right)$ y frecuencia respiratoria, para estimar el coeficiente de tolerancia al calor (CTC). El crecimiento de las corderas fue evaluado a través de las mediciones: peso vivo (PV), ganancia diaria de peso (GDP), largo corporal (LC), perímetro torácico (PT), altura a la cruz (AC), con las cuales se estimaron los índices de masa corporal 1 (IMC1) e índice de masa corporal 2 (IMC2). En cuanto a las variables climáticas, la TAmax y HR promedio resultaron mayores para SOMBRA que para $\mathrm{SOL}$, en $1,3^{\circ} \mathrm{C}$ y $8,1 \%$, respectivamente $(\mathrm{p}<0,01)$, debido tal vez al material usado como techo (lona) que formaba un microclima debajo del mismo que incrementaba estas variables. Sin embargo, la lona redujo en aproximadamente $90 \%$ la incidencia de RS en las áreas sombreadas durante las horas del día. En cuanto al ITH resultó superior para SOMBRA $(74,5)$ que para $\mathrm{SOL}(73,5)$, siendo ambos valores que se asocian con alerta o inicio de estrés, sin embargo los animales que pastorearon con SOMBRA obtuvieron niveles inferiores de CTC $(p<0,01) ; 3,21$ en SOMBRA vs 3,51 en SOL. En cuanto al crecimiento de las corderas, los resultados arrojaron que el uso de SOMBRA en los potreros no afectó el crecimiento de los animales, ya que no hubo efecto de tratamiento sobre PV, GDP, LC, AC, IMC1 e IMC2. Los valores promedios para PV fueron 14,59 y $15,10 \mathrm{~kg}$; mientras que las GDP resultaron de 80,8 y $82,6 \mathrm{~g} / \mathrm{d}$ para SOL y SOMBRA, respectivamente. En conclusión, la presencia de sombra artificial con techos de lona durante la época lluviosa produce incremento de HR, generando a su vez condiciones de estrés aún bajo SOMBRA, sin embargo, pudiera mitigar el efecto de la TA por reducción del calor extra-calórico en el animal debido a la disminución de la carga radiante. Asimismo, la presencia de SOMBRA en el potrero no redundó en un mejor crecimiento de las corderas, tal vez debido a lo breve de la experiencia.

\section{Thermal comfort and growth of West African lambs grazing with and without access to artificial shade}

\section{SUMMARY}

In order to evaluate the effect of the presence or absence of shade during grazing on thermal comfort and growth of West African crossbred lambs, a study was carried out using 24 lambs $13.08 \pm 3.0 \mathrm{~kg}$ live weight, randomly assigned to two treatments: SHADE: grazing star grass (Cynodon nlemfluensis) with voluntary access to artificial shade or shelter of $36 \mathrm{~m}^{2}$ ( $3 \mathrm{~m}^{2}$ per animal) and SUN: grazing star grass without availability of natural or artificial shade. The experiment was performed in the Sheep Unit of the Agronomy School - Universidad Central de Venezuela. The animals were grazing on two paddocks of star grass during 6 weeks. Climate measurements were take using two portable weather stations (one for each paddock and for SHADE was placed under the shelter), recording temperature $\left(\mathrm{TA},{ }^{\circ} \mathrm{C}\right)$, relative humidity $(\mathrm{HR}, \%)$ and solar radiation $\left(\mathrm{RS}, \mathrm{w} / \mathrm{m}^{2}\right)$, also some indexes were estimated: index of temperature and humidity or ITH and the coefficient of heat tolerance or CTC. The growth of lambs was assessed through weekly measurements of body weight (BW), for the estimation of daily weight gain (GDP), body length (LC), chest circumference (PT), wither height $(A C)$, through which the body mass index 1 (IMC1) and body mass index 2 (IMC2) were estimated. As results for climatic variables, TA and HR were higher for SHADE than 
for SUN, $1.3^{\circ} \mathrm{C}$ and $8.1 \%$, respectively $(p<0.01)$, perhaps due to the material used as a roof or shelter forming a microclimate under it that increased these variables. However, the shelter decreased approximately $90 \%$ incidence of RS in shaded areas during daylight hours. As the ITH was superior to SHADE (73.5) than SUN (74.5), both values are associated with stress start. However, CTC values for animals grazing with SHADE were lower $(p<0.01) ; 3.21$ vs 3.51 . Results also showed that the use of SHADE in the paddock did not affect the growth of the animals, since there was no effect of treatment on BW, GDP, $L C, A C$ and $I M C 1$ and IMC2. For PV the mean values were 14.59 and $15.10 \mathrm{~kg}$; while for GDP were 80.8 and 82.6 $\mathrm{g} / \mathrm{d}$ for SUN and SHADE, respectively. In conclusion, the presence of artificial shade with canvas roofs during the rainy season causes increased HR, generating stress conditions even in SHADE conditions. Also, the presence of SHADE in the paddock did not result in better growth of lambs, perhaps due to the short duration of the trial.

\section{INTRODUCCIÓN}

En los ovinos, al igual que otras especies de interés zootécnico, el estrés calórico tiene efectos marcados que involucran constantes fisiológicas y desempeño productivo. En vacunos, los efectos negativos del estrés calórico influyen la función reproductiva (Ponce, 1978; Wolfenson et al., 1997), producción de leche (Saravia et al., 2011), crecimiento e inmunidad (Nonaka et al., 2008). No obstante, este tipo de información no parece ser tan abundante en ovinos tropicales, en los que se reportan efectos del estrés calórico sobre el consumo y ganancia de peso (Filho et al., 2013), comportamiento (Santos et al., 2011) y resultados contradictorios desde el punto de vista reproductivo (Rodríguez et al., 2009).

Las zonas bajas de la franja tropical se caracterizan por presentar temperaturas ambientales (TA) y radiaciones solares (RS) elevadas la mayor parte del año, por lo que, los animales de producción pueden estar potencialmente afectados por condiciones de estrés calórico. Esto es particularmente cierto en el caso de los ovinos y otros rumiantes manejados en condiciones de pastoreo sin presencia de sombra en los potreros, situación que pudiera incrementar el calor corporal y/o reducir la eficiencia de los mecanismos de disipación de calor de los animales.

La raza West African (WA) es una de las más comunes en los rebaños ovinos en Venezuela. Su aceptación se basa principalmente en su relativa adaptabilidad y resistencia a nuestras condiciones ambientales y de manejo (Hurtado et al., 2003). En general, existe una relativa escasez de literatura científica relativa al efecto de las condiciones climáticas en los sistemas de producción con ovinos tropicales, especialmente en la raza West African, probablemente debido al carácter marginal que ha tenido la especie en el trópico Latinoamericano. Adicionalmente, bajo la premisa de mejorar el bienestar animal y debido a la importancia creciente de los ovinos como alternativa de producción animal en el país, intentar dilucidar el grado de confort térmico y/o tolerancia a las condiciones climáticas tropicales de estos animales a través de la relación entre los factores climáticos y las respuestas productivas de estos animales, reviste gran importancia.

Desde el punto de vista biometeorológico, el conocimiento del régimen térmico desarrollado por Thom (1959), que relaciona la TA y la humedad relativa (HR)
(Índice de Temperatura y Humedad, ITH), es el más difundido y practicado a la hora de establecer el nivel de confort animal (Martello et al., 2004). Adicionalmente, la utilización de índices que relacionen dos (2) o más variables fisiológicas pudieran ser utilizados como indicadores in vivo más eficientes del grado de estrés y/o tolerancia de los animales a condiciones estresantes. Dentro de estos índices destacan el Coeficiente de Tolerancia al Calor (CTC) (Souza et al., 2010), que relaciona la temperatura corporal (TC) y la frecuencia respiratoria (FR).

El objetivo de este trabajo fue determinar el efecto de la provisión de sombra en el potrero sobre el confort térmico y crecimiento de corderas West African en condiciones tropicales.

\section{MATERIAL Y MÉTODOS}

El estudio tuvo lugar en el Laboratorio Sección de Ovinos (LSO) del Instituto de Producción Animal, de la Facultad de Agronomía, Universidad Central de Venezuela. El Instituto de Producción Animal está ubicado a 432 msnm, en una región agroecológica descrita como bosque seco tropical (Holdridge, 1979), con una precipitación de 600-800 mm anuales, concentrada entre los meses de mayo a octubre. La TA promedio es $26,5^{\circ} \mathrm{C}$, con mínimas de 21,0 y máximas de $32,0^{\circ} \mathrm{C}$. HR es de alrededor de $60 \%$ en los meses secos y de $80 \%$ en los meses de lluvia. El experimento se realizó entre agosto y septiembre, meses de lluvia, y tuvo una duración de seis (6) semanas.

Se utilizaron hembras ovinas tropicales en crecimiento (de alto mestizaje WA), con peso y edad de $13,08 \pm 3,0 \mathrm{~kg}$ y 3-31/2 meses, respectivamente. El manejo de los animales fue el mismo que se realiza en forma habitual en el LSO: el rebaño se encontraba en condiciones de semi-estabulación, con pastoreo en potreros de pasto estrella (Cynodon nlemfluensis; 9,5\% proteína cruda) en las horas diurnas (08:00 a 16:00) y confinamiento en corrales el resto del tiempo. Las corderas fueron suplementadas diariamente con bloque mineral comercial y alimento concentrado a base de subproductos de cervecería (300 g/animal/día; 16\% de proteína cruda).

Bajo un diseño completamente al azar, 24 corderas fueron separadas en dos (2) tratamientos: a) SOMBRA $(n=12)$ : pastoreo continuo en potrero con acceso voluntario a sombra artificial brindada con cuatro (4) techos de lona color verde de 2,8 $\mathrm{m}$ de altura. Estos techos, de $3 \times 3 \mathrm{~m}$ cada uno, fueron colocados uno al lado del otro en posición este-oeste. La superficie total de sombra fue $36 \mathrm{~m}^{2}$, equivalente a $3 \mathrm{~m}^{2}$ por animal; b) SOL $(\mathrm{n}=12)$ : consistió en el pastoreo continuo en potrero sin disponibilidad sombra natural o artificial.

Se realizó la caracterización climática a través del registro horario de los factores climáticos TA $\left({ }^{\circ} \mathrm{C}\right), \mathrm{HR}$ $(\%)$ y radiación solar $(\mathrm{RS})\left(\mathrm{w} / \mathrm{m}^{2}\right)$; los mismos fueron obtenidos por medio de sensores electrónicos que estuvieron conectados a estaciones climáticas meteorológicas automáticas portátiles, una para el potrero con sombra y otra para el potrero sin sombra. En el caso del potrero con sombra, la estación se colocó debajo de los 
techos. La información climática fue almacenada en un datalogger y fue descargada semanalmente con el uso de un ordenador.

El crecimiento de las corderas fue evaluado a través de las siguientes mediciones y/o índices biométricos de estado corporal (IBEC):: peso vivo (PV, kg), largo corporal $(\mathrm{LC}, \mathrm{cm})$, perímetro torácico $(\mathrm{PT}, \mathrm{cm})$, altura de la cruz $(\mathrm{AC}, \mathrm{cm})$, índice de masa corporal 1 $(\mathrm{IMC} 1)=\mathrm{PV} /$ altura de la cruz $(\mathrm{AC})^{2}\left(\mathrm{~kg} / \mathrm{m}^{2}\right)$ e índice de masa corporal $2(\mathrm{IMC} 2)=\mathrm{PV} / \mathrm{AC}{ }^{\star} \mathrm{LC}\left(\mathrm{kg} / \mathrm{m}^{2}\right)$ (Khan y Khan, 2004). El PV (kg) fue obtenido mediante el pesaje matutino quincenal en romana de $400 \mathrm{~kg}$ de capacidad y $\pm 100 \mathrm{~g}$ de precisión; LC (cm) y AC (cm) fueron determinadas con una regla calibrada (precisión $\pm 1 \mathrm{~cm}$ ) y medidas desde la región de la cruz hasta la inserción de la cola y desde el plano de sustentación del individuo a la línea dorsal de la región de la cruz, respectivamente; el PT $(\mathrm{cm})$ fue determinado con cinta métrica (precisión $\pm 1 \mathrm{~cm}$ ) colocándola alrededor de la región del tórax.

La evaluación del confort térmico de los animales se realizó a través de dos (2) índices: 1) Índice de TA y HR (ITH), que relaciona los factores climáticos TA y HR (Thom, 1959) y, 2) el Coeficiente de Tolerancia al Calor (CTC) o Índice de Benezra que relaciona los índices fisiológicos TC y la FR. Las ecuaciones usadas en cada caso fueron las siguientes:

$\mathrm{ITH}=(1,8 \mathrm{TA}+32)-(0,55-0,55 \mathrm{HR} / 100) \times(1,8 \mathrm{TA}-26)$

Donde:

TA, es la temperatura ambiente $\left({ }^{\circ} \mathrm{C}\right) \mathrm{y}$

HR (\%), es la humedad relativa (Valtorta y Gallardo, 1996).

$$
\mathrm{CTC}=(\mathrm{TC} / 39)+(\mathrm{FR} / 23)
$$

Donde:

TC es la temperatura corporal $\left({ }^{\circ} \mathrm{C}\right) \mathrm{y}$

FR es frecuencia respiratoria (inspiraciones/min) (Souza et al., 2010).

Esta ecuación utiliza las medias de TC reportada para pequeños rumiantes (Medeiros y Vieira, 1997; Martins Junior et al., 2007; Pinto-Santini et al., 2014) y las medias de FR obtenidas para la mayoría de los animales de interés zootécnico (Medeiros y Vieira, 1997) esto, debido a la escasa literatura de valores de TC y FR en ovinos del tipo West African; cuanto el CTC es más próximo a dos (2), se considera que los animales son más tolerantes al calor.

Se calculó el ITH horario, obteniéndose promedios diarios, considerándose que valores entre 71 y 79 unidades, indican una condición de alerta, de acuerdo a lo señalado por la World Meteorological Organization (1989). Adicionalmente, se calculó el número de horas del día en que se presentaron $\mathrm{ITH} \geq 72$ (Valtorta et al., 2002). La TC se determinó con el uso de un termómetro digital Testo 110 y una sonda de inmersión/penetración, introducida durante 20 segundos a nivel del recto de cada uno de los animales a una profundidad de $5 \mathrm{~cm}$. La FR fue calculada observando las expansiones costo-abdominales durante 60 segundos. Tanto TC como FR fueron determinados quincenalmente, en dos (2) días fijos (A y B), durante dos (2) momentos diferentes del día (AM: entre las 07:30 y 09:00 horas, en manga de trabajo previo al traslado a los potreros; PM: entre las 15:00 y 17:00 horas, en corrales portátiles dentro del potrero con descanso de 10 minutos antes de la medición y respectando la condición de SOMBRA y SOL). Toda la metodología aquí planteada cumple con la Norma de la Comisión de Bioética y Bioseguridad de la Facultad de Agronomía de la Universidad Central de Venezuela.

El análisis de los datos se realizó a través del análisis de varianza para medidas repetidas en el tiempo. El modelo lineal incluía el efecto del tratamiento (SOL vs. SOMBRA), efecto de la semana de medición (para IBEC y CTC) y momento de medición (AM vs. PM; para (TC) y sus respectivas interacciones. Se aplicaron las pruebas de comparación de medias por Tukey-Kramer y/o Fisher's PLSD, según el caso, en las variables cuyas diferencias fueron significativas. El programa estadístico utilizado fue StatView versión 5,0 (SAS Institute Inc. Copyright, 1992-1998). Adicionalmente, se realizó un análisis de correlación de Pearson entre los IBEC.

\section{RESULTADOS Y DISCUSIÓN}

\section{Caracterización del AMBIENTE ClimÁtico}

En la tabla I, se observan los valores promedios ( $\pm \mathrm{DE})$, mínimos y máximos para TA, HR y RS en las

Tabla I. Valores medios, valores mínimos y valores máximos para la temperatura ambiente (TA), humedad relativa (HR) y radiación solar (RS) en las condiciones de sol y sombra durante el periodo experimental (Mean values, minimum and maximum values for the temperature (TA), relative humidity $(\mathrm{RH})$ and solar radiation (RS) under the conditions of SUN and SHADE during the experimental period).

\begin{tabular}{lcc}
\hline VARIABLE & SOL & SOMBRA \\
\hline \multicolumn{3}{c}{ Temperatura ambiente $\left({ }^{\circ} \mathrm{C}\right)$} \\
\hline TA (mean values) & $24,3 \pm 1,1$ & $24,4 \pm 1,0$ \\
Mínima & $19,5 \pm 0,9$ & $19,4 \pm 0,8$ \\
Máxima & $31,5 \pm 1,9^{\mathrm{b}}$ & $32,8 \pm 2,5^{\mathrm{a}}$ \\
\hline \multicolumn{3}{c}{ Humedad relativa $(\%)$} \\
\hline HR (mean values) & $77,8 \pm 4,2^{\mathrm{b}}$ & $85,9 \pm 5,1^{\mathrm{a}}$ \\
Mínima & $47,4 \pm 7,7^{\mathrm{b}}$ & $52,1 \pm 10,0^{\mathrm{a}}$ \\
Máxima & $90,9 \pm 0,5^{\mathrm{b}}$ & $100,0 \pm 0,0^{\mathrm{a}}$ \\
\hline \multicolumn{3}{c}{} \\
\hline RS (mean values) & Radiación Solar $\left(\mathrm{W} / \mathrm{m}^{2}\right)$ \\
Mínima & $299,7 \pm 67,4^{\mathrm{a}}$ & $29,9 \pm 20,0^{\mathrm{b}}$ \\
Máxima & $23,4 \pm 4^{\mathrm{a}}$ & $6,2 \pm 3,1^{\mathrm{b}}$ \\
& $716,1 \pm 144,0^{\mathrm{a}}$ & $54,4 \pm 17,9^{\mathrm{b}}$
\end{tabular}

TAmin $=$ valores medios de las TA mínimas diarias presentadas en el periodo experimental; TAmax= valores medios de las TA máximas diarias presentadas en el periodo experimental; $\mathrm{HRmin}=$ valores medios de la HR mínimas diarias presentadas en el periodo experimental; HRmax= valores medios de la HR máxima diaria presentadas en el periodo experimental; RSmin= valores medios de la RS mínima diaria durante las horas diurnas (07:00 a 18:00 horas) en el periodo experimental; RSmax= valores medios de la RS máxima diaria durante las horas diurnas (07:00 a 18:00 horas) en el periodo experimental. Letras diferentes en la misma fila indican diferencias significativas $(p \leq 0,01)$. 


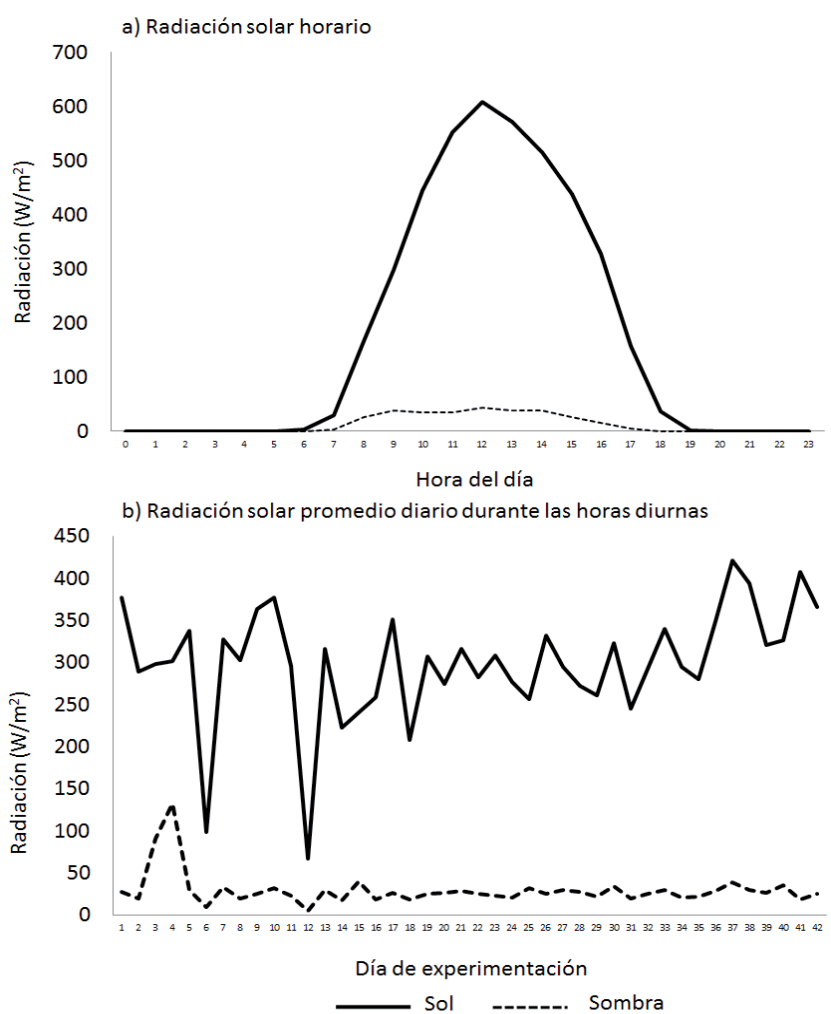

Figura 1. Radiación solar a lo largo del día (1a) y a lo largo del experimento (1b), durante las horas diurnas $\left(\mathrm{W} / \mathrm{m}^{2}\right)$ en SOL y SOMBRA (Solar radiation during of day (1a) and experimental period (1b) in SUN and SHADOW).

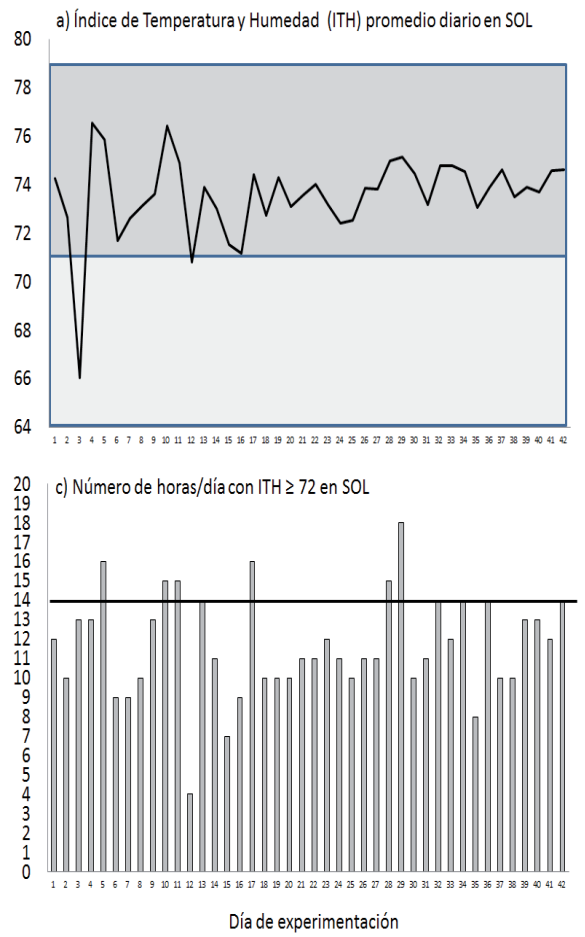

dos (2) condiciones evaluadas. Se observan valores similares entre tratamientos para TA y TAmin $(p>0,05)$. Sin embargo, para TAmax y HR promedio se presentó un incremento de $1,3^{\circ} \mathrm{C}$ y $8,1 \%$ en SOMBRA respecto a SOL $(p \leq 0,01)$. Las TAmax se presentaron entre las 12:00 a 15:00 h y 11:00 a 14:00 h y las TAmin entre las 05:00 a 07:00 h y 04:00 a 06:00 h, en SOL y SOMBRA, respectivamente. La HRmin se presentaron durante las horas de máxima TA y la HRmax durante las horas de la madrugada (00:00 a 06:00 horas), en ambos tratamientos. La correlación entre la TA y HR fue alta y negativa $(r=-0,914$ y $-0,916$ para SOMBRA y SOL, respectivamente; $\mathrm{p} \leq 0,01)$, situación que se ajusta a la relación ambiental tropical descrita por Oliveros et al. (2008) para el bosque seco tropical en la región, en la que se observa que la HR disminuye en las horas del día en los que se presenta la mayor TA.

El techo de lona redujo en aproximadamente $90 \%$ la incidencia de la RS en la zona sombreada durante las horas diurnas. Las horas de mayor RS se presentaron entre las 12:00 y las 14:00 horas, siendo que a las 13:00 horas la misma alcanzó, en promedio, $608,5 \mathrm{~W} / \mathrm{m}^{2}$ en el caso de SOL y $42,9 \mathrm{~W} / \mathrm{m}^{2}$ en SOMBRA $(\mathrm{p} \leq 0,01)$ (figura 1a). Por día, se evidencia que durante toda la fase experimental, el potrero del tratamiento SOL presentó niveles de RS superiores en comparación con la condición SOMBRA (figura 1b).

La precipitación total $(\mathrm{mm})$ del mes de los meses de agosto y septiembre en la zona de estudio fue de 257 y 190, respectivamente (CENIAP), siendo éstos los

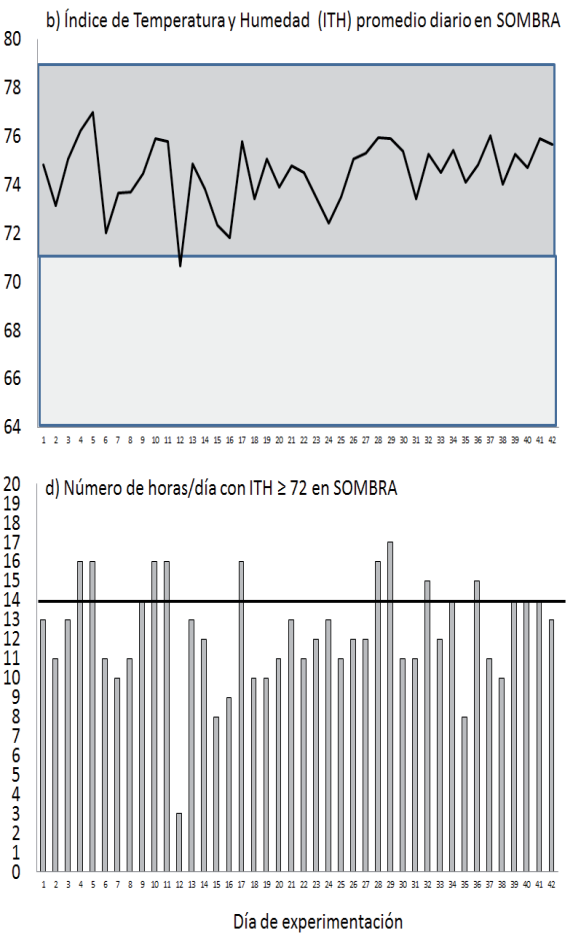

Figura 2. Índice de temperatura y humedad (ITH) promedio diario (2a y 2b) y número de horas en los que se presentaron ITH $\geq 72$ (2c y 2d) en los tratamientos de SOL y SOMBRA. La zona gris clara indica una condición normal (sin estrés) y la zona gris oscura una condición de alerta o inicio de estrés en los animales (World Metereological Organization, 1989). Más de 14 horas al día por tres (3) o más días consecutivos podrían significar una condición de estrés en los animales (Valtorta et al., 2002) (Index of temperatura and humidity (ITH) by day and number of hours with ITH $\geq 72$ (2c y $2 \mathrm{~d}$ ) in SUN and SHADOW. The light gray area indicates a normal condition (without stress) and the dark gray area an alert condition by stress in animals (World Metereological Organization, 1989). More than 14 hours a day for three (3) or more consecutive days could mean a condition of stress in animals). 
meses de mayor precipitación para ese año, situación que pudo causar incrementos en la HR en la zona de estudio y particularmente en el microclima debajo del toldo, el cual, debido a sus características, acumuló humedad. Durante los 42 días de evaluación se presentaron un total de 22 días de lluvia.

\section{INDICES DE CONFORT TÉRMICO}

En la figura 2 puede observarse los valores diarios (figura 2a y 2b) de ITH y las horas del día en que se presenta la condición de ITH $\geq 72$ (figura 2 c y 2d). El ITH promedio (unidades) fue superior en la condición de SOMBRA $(74,5 \pm 1,7)$ en comparación con la condición de SOL $(73,5 \pm 1,3)(p \leq 0,01)$. Las horas del día en que el ITH fue $\geq$ a 72 unidades fue, en promedio, de $11,7 \pm 2,6$ para SOL y $12,3 \pm 2,7$ para SOMBRA, sin diferencias significativas $(p>0,05)$, valores éstos cercanos a la condición de $14 \mathrm{~h}$ con ITH $\geq 72$ reportado por Valtorta et al. (2002). La situación ambiental en ambos tratamientos, desde el punto de vista del ITH, se asocia con condiciones de alerta y/o inicio de estrés. En ovinos tropicales el punto de quiebre del ITH no ha sido reportado. Sin embargo, Marai et al. (2007) reporta que en ovinos ITH $<82$ representan ausencia de estrés. En base a lo anterior, posiblemente, las condiciones ambientales presentadas no necesariamente estarían afectando en forma importante a los animales, lo que no descarta la condición de alerta. Neves et al. (2009) evaluaron los niveles críticos de confort térmico en ovinos de la raza Santa Inés a través de estudios de correlación entre variables climáticas y respuestas animales en términos de TC y FR. El valor crítico del índice de confort térmico (ICT) establecido, considerando la FR de los animales, fue 38; considerando la TC, los autores refieren niveles de 46,3; 45,5 y 44,4 para animales Santa Inés con coloración de pelo blanco, castaño y negro, respectivamente. A pesar del importante aporte realizado por Neves et al. (2009), el cálculo de los valores ICT requiere equipos especiales que no siempre son disponibles a nivel experimental y/o finca, tal es el caso de la determinación de la temperatura de globo negro, presión parcial de vapor y la velocidad del viento, lo que pudiera limitar un poco su uso en comparación con índices más sencillos y de uso masivo como el ITH.

En la tabla II se presentan los valores promedios quincenales del CTC durante la fase experimental. Los animales bajo SOL presentaron valores superiores de CTC en comparación con los de SOMBRA $(p \leq 0,01)$. Asimismo, la hora de medición influyó en la respuesta
Tabla II. Índices de masa corporal (IMC) en ovinos en crecimiento sometidos a pastoreo con $y$ sin presencia de sombra en los potreros (Body mass index (BMI) in growing lambs subjected to pasture with and without presence of shadows).

\begin{tabular}{lccc}
\hline SEMANA & SOL & SOMBRA & Valores medios \\
\hline \multicolumn{4}{c}{ IMC1 $=$ Peso vivo/Altura de la cruz ${ }^{2}$} \\
\hline 1 & $52,1 \pm 4,6$ & $51,3 \pm 6,7$ & $51,7 \pm 5,7^{\mathrm{b}}$ \\
2 & $57,2 \pm 5,1$ & $58,0 \pm 8,8$ & $57,6 \pm 7,2^{\mathrm{a}}$ \\
4 & $57,9 \pm 5,7$ & $59,3 \pm 6,9$ & $58,7 \pm 6,3^{\mathrm{a}}$ \\
6 & $58,3 \pm 6,8$ & $59,3 \pm 7,5$ & $58,8 \pm 7,0^{\mathrm{a}}$ \\
Valores medios & $56,4 \pm 6,0$ & $57,0 \pm 8,0$ & - \\
\hline
\end{tabular}

IMC2= Peso vivo/Altura de la cruz*Largo corporal

\begin{tabular}{lccc}
\hline 1 & $59,7 \pm 6,8$ & $59,4 \pm 9,5$ & $59,6 \pm 8,1^{\text {b }}$ \\
2 & $65,3 \pm 6,3$ & $65,2 \pm 9,3$ & $65,2 \pm 7,9^{\text {ab }}$ \\
4 & $65,9 \pm 6,6$ & $66,6 \pm 7,9$ & $66,3 \pm 7,2^{\text {a }}$ \\
6 & $66,4 \pm 6,3$ & $67,7 \pm 8,8$ & $67,1 \pm 7,5^{\text {a }}$ \\
Valores medios & $64,4 \pm 6,8$ & $64,8 \pm 9,1$ & - \\
\hline
\end{tabular}

Letras diferentes en la misma columna indican diferencias significativas $(p \leq 0,05)$.

de los animales, siendo que en la AM se presentaron CTC inferiores a los PM, para ambos tratamientos $(\mathrm{p} \leq 0,01)$. Sin embargo, los valores de CTC fueron superiores a dos (2) en ambos tratamientos, mostrando una posible situación de inicio de estrés en los dos casos, según reportes de Souza et al. (2010). La exposición de los animales a la RS directa elevó en 39\% en el valor de CTC en vacas lecheras. El CTC antes, inmediatamente y una hora despues de la exposición solar directa fue: 3,68; 5,13 y 3,80 ( $\mathrm{p}<0,05$; Souza et al., 2010), valores estos reflejos de que la exposición al sol provocó un estrés alto en vacas. Para el caso concreto de los ovinos de esta experiencia, los valores promedios de CTC ligeramente superiores a 3 (tabla III) se asociaron con incrementos de aproximadamente $0,5^{\circ} \mathrm{C}$ en la TR y la duplicación en la FR típicas de los pequeños rumiantes.

Al comparar los valores de ITH y CTC presentados en esta experiencia, destaca que, a pesar de que en SOMBRA el ITH fue superior a SOL $(p \leq 0,01)$, los animales que pastorearon en el potrero con acceso a sombra artificial, presentaron niveles inferiores de CTC $(p \leq 0,01)$. Estudios preliminares realizados en el mes de mayor RS histórica en la región central de Venezuela $\left(10,25^{\circ} \mathrm{LN}\right.$ y $\left.67,65^{\circ} \mathrm{LO}\right)$, con hembras WA durante el

Tabla III. Coeficiente de tolerancia al calor (CTC) por semana y hora del día en las condiciones de SOL y SOMBRA (Coefficient of heat tolerance (CTC) by week and hour of day in SUN and SHADOW).

\begin{tabular}{|c|c|c|c|c|c|}
\hline \multirow[t]{2}{*}{ SEMANA } & \multicolumn{2}{|c|}{ SOL } & \multicolumn{2}{|c|}{ SOMBRA } & \multirow[b]{2}{*}{ Valores medios } \\
\hline & AM & PM & AM & PM & \\
\hline 0 & $3,32 \pm 0,48$ & $5,33 \pm 1,034$ & $3,03 \pm 0,50$ & $4,75 \pm 0,82$ & $4,09 \pm 1,20^{c}$ \\
\hline 2 & $3,16 \pm 0,63$ & $2,80 \pm 0,41$ & $2,93 \pm 0,38$ & $2,72 \pm 0,27$ & $2,90 \pm 0,46^{a}$ \\
\hline 4 & $3,07 \pm 0,56$ & $3,74 \pm 0,90$ & $2,77 \pm 0,45$ & $3,59 \pm 0,74$ & $3,29 \pm 0,78^{b}$ \\
\hline 6 & $3,24 \pm 0,54$ & $3,35 \pm 0,60$ & $2,86 \pm 0,38$ & $3,03 \pm 0,37$ & $3,11 \pm 0,51^{\mathrm{ab}}$ \\
\hline Valores medios hora & $3,20 \pm 0,55^{a}$ & $3,82 \pm 1,21^{b}$ & $2,90 \pm 0,43^{a}$ & $3,53 \pm 0,98^{b}$ & \\
\hline Valores medios tratamiento & \multicolumn{2}{|c|}{$3,51 \pm 0,99^{a}$} & \multicolumn{2}{|c|}{$3,21 \pm 0,81^{b}$} & \\
\hline
\end{tabular}

Letras diferentes entre columnas y/o fila indican diferencias significativas $(p \leq 0,01)$. 
crecimiento, demostraron el mejor confort térmico de los animales pastoreando en potreros con sombra natural (Pinto-Santini et al., 2011). En general, los índices fisiológicos de los animales en condiciones tropicales se incrementan en las horas de la tarde. Santos et al. (2006) reportan valores AM vs. PM de TC en ovinos de $39,0-39,2$ a $39,6-39,7^{\circ} \mathrm{C}$ y FR $47,3-56,0$ a $83,3-115$, respectivamente. Similares resultados son presentados por Quesada et al. (2001).

Los factores ambientales evaluados demostraron incrementos en la TAmax, HR, HRmin y HRmax en la condición de SOMBRA, indicativo de que el techo utilizado favoreció la acumulación de humedad debajo del mismo, debido, entre otras, a la elevada precipitación presentada durante la fase experimental y al tipo de material (lona) utilizado para la elaboración de los mismos. Sin embargo, el techado fue muy eficiente a la hora de promover un espacio sombreado para los animales. El índice de confort térmico asociado a las variables ambientales (ITH), fue la mayor parte del tiempo $\geq$ a 72 en ambas condiciones ambientales. Es decir, las condiciones climáticas presentadas pueden ser consideradas como de alerta por estrés, aún en ovinos. Los mayores valores de ITH se presentaron en la condición de SOMBRA. Los valores de CTC fueron superiores a dos (2) en ambos tratamientos y en las diferentes semanas y horas de medición evaluadas, situación refleja de las condiciones ambientales. Sin embargo, desde el punto de vista de la respuesta animal, medida a través del CTC, se observaron menores valores en la condición de SOMBRA, lo que pudiera traducirse como un mejor confort en los animales con acceso al techado, es decir, a pesar de las condiciones climáticas presentadas en ambos tratamientos, los animales con acceso a sombra en los potreros lograron mitigar un poco la situación reflejada con el ITH, posiblemente por una disminución de la carga radiante.

\section{ÍNDICES BIOMÉTRICOS DE ESTADO CORPORAL (IBEC)}

El uso de sombra en los potreros no afectó el crecimiento de los animales dado que, no se presentó efecto del tratamiento sobre PV, LC, AC, IMC1 e IMC2 ( $p>0,05)$. Los valores promedios para PV fueron $14,6 \pm 3,2$ para SOL y $15,1 \pm 3,7$ para SOMBRA $(\mathrm{p}>0,05)$; la GDP para SOL y SOMBRA fue $80,8 \pm 9,2$ y $82,6 \pm 12,5$ respectivamente, con diferencias de peso entre el final y el inicio del experimento de $(\mathrm{kg}) 3,7 \pm 1,2$ para SOL y $3,7 \pm 0,7$ para SOMBRA $(p>0,05)$ (tabla IV). Las GDP fueron similares a las reportadas en otras condiciones de explotación semi-extensiva en Venezuela, tal es el caso de los Llanos Occidentales (Zambrano et al., 1999) y Región Occidental en la Cuenca del Lago de Maracaibo (Goicochea et al., 1980), e inferiores a las reportadas en corderas Pelibuey en México (192 \pm 7 g/d; CórdovaPascual et al., 2009).

La edad promedio de los animales fue $90 \mathrm{~d}$ al inicio y $132 \mathrm{~d}$ al final del experimento. Cuando comparamos los PV obtenidos en esta experiencia con los de otros reportes de la literatura a edades similares, los mismos son inferiores. En tal sentido, Santos et al. (2001), reportó pesos promedio en animales jóvenes de la raza Santa Inés de $59,9 \pm 13,3 ; 35,0 \pm 12,6$ y $30,4 \pm 12,8$ para niveles de alimentación alto, medio y bajo, respectivamente
Tabla II. Peso vivo y ganancias diarias de peso promedios quincenales durante la fase experimental (Body weight and daily weight gain during experimental period).

\begin{tabular}{lccc}
\hline SEMANA & SOL & SOMBRA & Valores medios \\
\hline \multicolumn{4}{c}{ Peso vivo $(\mathrm{kg} \pm \mathrm{DE})$} \\
\hline 1 & $12,25 \pm 2,66$ & $12,83 \pm 2,93$ & $12,44 \pm 2,62^{\mathrm{b}}$ \\
2 & $14,02 \pm 2,88$ & $14,55 \pm 3,15$ & $14,62 \pm 3,19^{\mathrm{a}}$ \\
4 & $14,76 \pm 3,17$ & $15,57 \pm 2,99$ & $15,46 \pm 3,26^{\mathrm{a}}$ \\
6 & $16,02 \pm 3,13$ & $16,55 \pm 3,17$ & $16,81 \pm 3,34^{\mathrm{a}}$ \\
Valores medios & $14,59 \pm 3,15$ & $15,10 \pm 3,70$ & - \\
\hline
\end{tabular}

Ganancias de peso vivo (g/día DE)

\begin{tabular}{lrrr}
\hline 1 a la 2 & $126,78 \pm 25,82$ & $123,01 \pm 47,21$ & $120,63 \pm 40,47^{\mathrm{a}}$ \\
2 a la 4 & $52,67 \pm 51,49$ & $73,01 \pm 55,18$ & $60,00 \pm 50,42^{\mathrm{b}}$ \\
4 a la 6 & $90,17 \pm 23,51$ & $69,84 \pm 95,55$ & $84,58 \pm 71,20^{\text {ab }}$ \\
Valores medios & $85,98 \pm 46,92$ & $88,47 \pm 70,07$ & -
\end{tabular}

Letras diferentes en la misma columna indican diferencias significativas $(p \leq 0,05)$.

$(\mathrm{p} \leq 0,01)$. Similarmente, Texeira et al. (2012) reportan pesos de $17,0 \pm 0,8$ en animales pastoreando pasturas cultivadas y $16,4 \pm 0,7$ en animales pastoreando en pasturas nativas. Con relación a la semana de experimentación, tanto para PV como para GDP, se observaron diferencias altamente significativas $(p \leq 0,01)$, situación que evidencia que, a pesar de la baja tasa de crecimiento presentada, los animales incrementaron su PV a lo largo del experimento.

En la tabla V, pueden observarse los valores promedios quincenales de AC, LC y PT durante la fase experimental. No se presentaron diferencias significativas entre tratamientos y, con excepción del PT, hubo efecto de la semana de experimentación $(p \leq 0,05)$, es decir, los animales incrementaron su talla y longitud a lo largo del experimento. Similares resultados fueron presentados para los IMC1 e IMC2 (tabla II).

Son pocos los trabajos realizados en Venezuela donde se reporten variables como AC, LC y PT en animales WA. Sin embargo, al comparar los resultados con los estudios realizados en Brasil con la raza Santa Inés, producto del cruce entre WA y Bergamasca, se evidencia el menor tamaño del mestizo WA venezolano. En tal sentido, observamos valores $(\mathrm{cm})$ de $53,1 \pm 3,2$ para AC y $46,4 \pm 2,5$ para LC en los animales de esta experiencia en comparación con rangos entre 69,1-73,8 para AC y 67,2-73,5 de LC en la raza Santa Inés (Santos et al., 2001). Hurtado et al. (2003), reportan los siguientes IBEC promedios en corderos WA y Barbados Barriga Negra (BBN) a los 90 días de edad: $\mathrm{PV}=10,77 \mathrm{~kg} ; \mathrm{PT}=50,15 \mathrm{~cm}$; profundidad toráci$\mathrm{ca}=20,07 \mathrm{~cm} ; \mathrm{AC}=47,74 \mathrm{~cm}$ y $\mathrm{LC}=42,06 \mathrm{~cm}$ y señalan que los corderos provenientes de partos simples y los de la raza BBN presentan una talla y peso mayor que los corderos provenientes de partos dobles y los de la raza WA, con diferencias significativas $(p<0,05)$ en todas las variables estudiadas.

Respecto a los IMC evaluados, los resultados son similares a los reportados por Costa Junior et al. (2006), los cuales reportaron menores aumentos en el índice 
Tabla V. Altura de la cruz (a), largo corporal (b) y perímetro torácico (c) en ovinos sometidos a pastoreo con y sin presencia de sombra artificial en el potrero (Wither heigth, body length and chest circumference of the sheep in grazing with or without artificial shadow).

\begin{tabular}{|c|c|c|c|}
\hline SEMANA & SOL & SOMBRA & Valores medios \\
\hline \multicolumn{4}{|c|}{ Altura de la cruz $(\mathrm{cm})$} \\
\hline 1 & $48,2 \pm 3,7$ & $49,3 \pm 3,0$ & $48,8 \pm 3,3^{b}$ \\
\hline 2 & $49,9 \pm 3,8$ & $50,0 \pm 2,8$ & $50,0 \pm 3,2^{b}$ \\
\hline 4 & $50,8 \pm 4,0$ & $51,1 \pm 3,3$ & $51,0 \pm 3,5^{\mathrm{ab}}$ \\
\hline 6 & $52,7 \pm 3,0$ & $53,5 \pm 3,4$ & $53,1 \pm 3,2^{a}$ \\
\hline Valores medios & $50,5 \pm 3,8$ & $51,0 \pm 3,4$ & - \\
\hline \multicolumn{4}{|c|}{ Largo corporal (cm) } \\
\hline 1 & $42,1 \pm 2,6$ & $43,2 \pm 2,9$ & $42,7 \pm 2,7^{c}$ \\
\hline 2 & $43,7 \pm 2,6$ & $44,5 \pm 2,8$ & $44,1 \pm 2,7^{\mathrm{cb}}$ \\
\hline 4 & $44,5 \pm 2,5$ & $45,5 \pm 2,8$ & $45,1 \pm 2,6^{\mathrm{ab}}$ \\
\hline 6 & $46,2 \pm 2,6$ & $46,7 \pm 2,5$ & $46,4 \pm 2,5^{\mathrm{a}}$ \\
\hline Valores medios & $44,2 \pm 2,9$ & $45,0 \pm 2,9$ & - \\
\hline \multicolumn{4}{|c|}{ Perímetro torácico $(\mathrm{cm})$} \\
\hline 1 & $60,9 \pm 3,5$ & $62,6 \pm 3,8$ & $61,8 \pm 3,7$ \\
\hline 2 & $60,7 \pm 4,6$ & $61,2 \pm 4,3$ & $61,0 \pm 4,3$ \\
\hline 4 & $61,1 \pm 3,9$ & $62,1 \pm 4,2$ & $61,6 \pm 4,0$ \\
\hline 6 & $61,2 \pm 4,5$ & $62,7 \pm 4,6$ & $62,0 \pm 4,5$ \\
\hline Valores medios & $61,0 \pm 4,0$ & $62,1 \pm 4,1$ & - \\
\hline
\end{tabular}

Letras diferentes en la misma columna indican diferencias significativas $(p \leq 0,05)$.

de capacidad corporal que relaciona el PV con el LC. Estudios de alometría en corderos Santa Inés demostraron que existió crecimiento alométrico positivo entre el PV y la AC, crecimiento isométrico entre el PV y el PT y crecimiento alométrico negativo entre el PV y el LC, es decir, estos animales durante sus primeros meses de edad, priorizaron su crecimiento en talla (AC), PV y por último, LC (Koritiaki et al., 2012), situación está similar a la presentada en esta experiencia. La correlación entre todos los IBEC fue alta y positiva. La mayor correlación se presentó entre el PV-AC ( $\mathrm{r}=0,90$ y 0,89, para SOL y SOMBRA, respectivamente), seguida por PV-LC ( $r=0,86$ en SOL y $r=0,88$ en SOMBRA). La relación entre el PV-IMC1 y el PV-IMC2, en SOMBRA fue 0,90 y 0,92 y en SOL de 0,79 y 0,89 , respectivamente. En general, la presencia de sombra en el potrero no mejoró los índices de crecimiento de los animales. Los IBEC demostraron que las hembras WA en la etapa postdesdete, bajo las condiciones experimentales y de manejo del ensayo, crecen a una tasa de media a baja priorizando el crecimiento de su tejido óseo, siendo más altas (talla) que largas.

\section{CONCLUSIÓN}

Definición de nivel de ITH que pudiera indicar una condición de estrés en ovinos de pelo bajo condiciones tropicales no han sido reportados, sin embargo, a pesar de la adaptación del West African al trópico, los índices de confort térmico evaluados, tanto a nivel climático como a nivel de respuesta fisiológica, demuestran condiciones ambientales que pudieran considerarse como de alerta por estrés calórico aún en este tipo de animales. La presencia de sombra en el potrero, debido a la alta RS presentada en la zona, mitigó un poco el efecto de la alta TA presentada durante las horas del día, probablemente debido al mecanismo de reducción del calor extra-calórico provocado por la disminución de la carga radiante. El comportamiento productivo de los animales no se vio afectado por la presencia de sombra en el potrero; posiblemente sea necesaria una experiencia de mayor duración para poder evaluar apropiadamente estas variables. El uso de sombra en animales a pastoreo bajo condiciones tropicales y/o clima cálido y sus implicaciones en la mejora del confort de los animales debe ser promovido, así mismo, es necesario continuar los estudios relacionados con la obtención de índices que permitan evaluar en forma rápida el inicio de una situación de alerta o inicio de estrés en ovinos bajo condiciones tropicales.

\section{AGRADECIMIENTOS}

Este trabajo es parte del proyecto de grupo PG-018308-2011/1, financiado por el Consejo de Desarrollo Científico y Humanístico de la Universidad Central de Venezuela.

\section{BIBLIOGRAFÍA}

Avendaño, R. L.; Álvarez, F. D.; Molina, L.; Saucedo, J. S. y Correa, A. 2004. Engorda de corderos Pelibuey y sus cruzas con Dorper y Katahdin bajo condiciones de estrés calórico. Memorias del XXVIII Congreso Nacional de Buiatría. Michoacán. México. pp. 10-13.

Costa Júnior, G.; Campelo, J. E.; Azevédo, D.M.M.; Filho, R. M.; Cavalcante, R. R.; Lopes, J. B. e Oliveira, M.E. 2006. Caracterização morfométrica de ovinos da raça Santa Inês criados nas microrregiões de Teresina e Campo Maior, Piauí. Rev Bras Zootecn, 35: 2260-2267.

Córdova-Pascual, A.; Oliva-Hernández, J.; Sánchez-Hernández, D.; Torres-Hernández, G.; Suárez-Oporta, M.E. y Hinojosa-Cuéllar, J.A. 1999. Crecimiento postdestete y eficiencia reproductiva de corderas Pelibuey con un sistema de alimentación intensiva. Arch Med Vet, 41: 205-212.

Filho, F.P.; Azevedo, M.; Costa, L.A.; Lana, A.M.; Ferreira, M. and Batista, A. 2013. Heat tolerance index, food intake and weigth gain of Santa Ines sheep in the agreste region of Pernambuco State, Brazil. Acta Scientiarum. Anim Sci, 35: 307-310.

Goicochea, J.; González, C.; Perozo, F. y Rincón, E. 1980. Peso al nacimiento y crecimiento en corderos West African. (Resumen y Tablas). VI Seminario Nacional de Ovinos y Caprinos. San Cristóbal, Venezuela. p. 58.

Holdrige, L. 1979. Ecología basada en zonas de vida. IICA. San José. Costa Rica. 12 pp.

Hurtado, A.; Salvador, A.; Morantes, M. y Colmenares, O. 2003. Relación entre el peso corporal, medidas corporales y edad en corderos de raza West African y Barbados Barriga Negra desde el nacimiento al destete. Rev Fac Cs Vets, 44: 145-155.

Khan, A and Khan, A. 2004. Prevalence and etiology of obesity. An overwiew. Pakistan J Nutr, 3: 14-25.

Koritiaki, N.A.; Riberiro, E.L.; Costantino, C.; Fernandes Junior, F. e Grandis, F. 2012. Estudo do crescimento alométrico das medidas corporais de cordeiros da raça Santa Inês. Synergismus Scyentifica UTFPR. Pato Branco, 07: 1-3.

Marai, I.F.M.; El-Darawany, A.A.; Fadiel, A. and Abdel-Hafez, M.A.M. Physiological traits as affected by heat stress in sheep: a review. Small Ruminant Res, 71: 1-12. 
Martello, L.S.; Júnior, H.S.; Pinheiro, M.; Silva, S. e Júnior, L.R. 2004. Avaliação do microclima de instalações para gado de leite com diferentes recursos de climatização. Eng Agríc, 24: 263-273.

Martins Júnior, L.M.; Costa, A.P.R.; Azevedo, D.M.M.R.; Turco, S.H.N.; Campelo, J.E.G. e Muratori, M.C.S. 2007. Adaptabilidade de caprinos Boer e Anglo-Nubiana às condições climáticas do meio-norte do Brasil. Arch Zootec, 56: 103-113.

Medeiros, L.F.D. y Vieira, D.H. 1997. Bioclimatología Animal. Disponible en: http://www.iz.ufrri.br/zootecnia_draa/Biblioteca/Fernando/ Apostila\%20de\%20Bioclimatologia\%20l.pdf (15/01/2015).

Neves, M.L.M.; Azevedo, M.; Costa, L.A.B.; Guim, A.; Leite, A.M. e Chagas, J.C. 2009. Níveis críticos do índice de Conforto Térmico para ovinos da raça Santa Inês criados a pasto no agreste do Estado de Pernambuco. Acta Scientiarum. Anim Sci, 31: 169-175.

Nonaka, I. Takusari, N.; Tajima, K.; Suzuki, T.; Higuchi, K. and Kurihara, M. 2008. Effects of high environmental temperatures on physiological and nutritional status of prepubertal Holstein heifers. LivestSci, 1 13: 14-23.

Oliveros, Y.; Requena, F.; León, A.; Ostos, M.; Parra, R.; Marquina, J. y Bastianelli, D. 2008. Aplicación del índice del confort térmico como estimador de periodos críticos en cría de pollos de engorde. Zootecn Trop, 26: 531-537.

Pinto-Santini, L.; Ríos de Álvarez, L.; Oliveros, I.; Pigliacampo, A. y Chacón, T. 2014. Índices fisiológicos en corderas tipo West African con acceso voluntario a sombra artificial bajo condiciones de emergencia de calor leve. Livestock Res Rural Dev, 26. Artículo 207. http://www. Irrd.org/lrrd26/11/pint26207.html (15/01/2015).

Pinto, L.; Dávila, F.; Aparicio, A.; Rios, L.; Oliveros, Y. y Pineda, J. 2011. Confort térmico en ovejas tropicales sometidas a dos niveles de radiación solar. Memorias de la XXII Reunión ALPA. Montevideo. Uruguay. 24 al 26 octubre de 2011.

Ponce, H.R. 1978. Efectos de stress térmico sobre la fertilidad del ganado bovino. Cienc Vet, 2: 265-291.

Quesada, M.; Mc Manus, C. e F. Araujo, A. 2001. Tolerânciaa o calor de duas raças de ovinos deslanados no Distrito Federal. Rev Bras Zootecn, 30: 1021-1026.

Rodríguez, M.; Montaldo, H.H.; Balcázar, J.A. y Hernández, J. 2009. Niveles de progesterona sérica en ovejas Pelibuey y Suffolk sometidas a estrés térmico. Vet Méx, 40: 197-202.

Santos, J. R.; Souza, B.B.; Souza, W. H.; Cezar, M. F. e Tavares, G.P. 2006. Respostas fisiológicas e gradientes térmicos de ovinos das raças
Santa Inês, Morada Nova e de seus cruzamentoscom a raça Dorper às condições do semi-árido nordestino. Ciênc Agrotec, 30: 995-1001.

Santos, C.L.; Pérez, J.R.O.; Siquiera, E.R.; Muniz, J.A. e Bonagúrio, S. 2001. Crescimento alométrico dos tecidos ósseo, muscular e adiposo na carcaça de cordeiros Santa Inês e Bergamácia. Rev Bras Zootecn, 30: 493-498.

Saravia, C.; Astigarraga, L.; Van Lier, E. y Bentancur, O. 2011 . Impacto de las olas de calor en vacas lecheras en Salto (Uruguay). Agrociencia Uruguay, 15: 93-102.

Silanikove, N. 2000. Effects of heat stress on the welfare of extensively managed domestic ruminants. Livest Prod Sci, 67: 1-18.

Souza, B.B.; Oliveira, I.J.; Mellace, E.M.; Siqueira, R.F.; Zotti, C.A. e Garcia, P.R. 2010. Avaliação do ambiente físico promovido pelo sombreamento sobre o proceso termorregulatório em novilhas leiteiras. Agrop Cientif No Semi-Árido, 6: 59-65.

Souza, E.D.; Souza, B.B.; Souza, W.H.; Cezar, M.F.; Santos, J.R. e Tavares, G.P. 2005. Determinação dos parâmetros fisiológicos e gradient térmico de diferentes grupos genéticos de caprinos no semi-árido. Ciênc Agrotec, 29: 177-184.

Teixeira, M.C.; Villarroel, A.B.; Pereira, E.S.; Oliveira, S.M.; Albuquerque, I.A. e Mizubuti, I.Y. 2012. Curva de crescimento de cordeiros oriundos de três sistemas de produção na Região Nordeste do Brasil. Ciênc Agrár, 33: 2011-2018.

Thom, E.C. 1959. The discomfort index. Weatherwise, 12: 57-59.

Vallorta, S.E. y Gallardo, M.R. 1996. El estrés por calor en producción lechera. En: Instituto Nacional de Tecnología Agropecuaria (Ed.). Miscelánea, 81: 173-185.

Valtorta, S.E.; Leva, P.; E. Gallardo, M.R. and Scarpatti, O.E. 2002. Milk production responses during heat waves events in Argentina. In: American Meteorological Society (Ed.). Conference on Biometeorology and Aerobiology (15th) International Congress on Biometeorology (16th). Kansas City. Mo. Boston. pp. 98-101.

Wolfenson, D.; Lew, B.J.; Thatcher, W.W.; Graber, Y. and Meidan, R. 1997. Seasonal and acute heat stress effects on steroid production by dominant follicles in cows. Anim Reprod Sci, 47: 9-19.

World Meteorological Organization. 1989. Animal Health and Production at Extremes of Weather. Technical Note N ${ }^{\circ} 191$. Ginebra. Suiza.

Zambrano, C.; Ciria, J. y Asenjo, M. 1999. Comportamiento productivo del ovino West African en los llanos occidentales de Venezuela. II. Crecimiento postdestete. Producción Latinoamericana. XXIV: Comunicación 5 . 